\title{
Contrast-enhanced Ultrasonography: A New Strategy to Confirm Cervical Pregnancy
}

\author{
Qingyun Song, MD ${ }^{a, b}$, Sha Hu ${ }^{a, b}$, Hong Luo, MD ${ }^{a, b}$, Taizhu Yang ${ }^{a, b}$, Qianqian Gao, MD ${ }^{a, b}$, Fan Yang, MD ${ }^{a, b, *}$ \\ ${ }^{a}$ Department of Ultrasonography, West China Second University Hospital, Sichuan University; ${ }^{b}$ Key Laboratory of Obstetric \& \\ Gynecologic and Pediatric Diseases and Birth Defects of Ministry of Education, Chengdu, Sichuan Province 610041, China \\ Received May 15, 2019; revision received July 14; accepted July 15.
}

\begin{abstract}
Cervical pregnancy is a rare variety of ectopic pregnancy. The success of conservative treatment for cervical pregnancy depends on early and accurate diagnosis of ultrasound. We found Contrast-enhanced ultrasonography (CEUS) to be an accurate diagnosis approach for cervical pregnancy. In cervical pregnancy, CEUS showed rapid enhancement of the wall of the gestational sac during the early enhancement phase with the performance of hyper-enhanced. Enhancement sustained at a high level of intensity through the early enhancement phase, with the sign of doughnut-shaped in the wall of the gestational sac, and washed out slowly during the late enhancement phase. While the wall of the gestational sac showed no enhancement during the whole phase in aborting intrauterine pregnancy residing in the cervical canal.
\end{abstract}

Key words: Contrast-enhanced ultrasound; Sonography; Cervical pregnancy; Inevitable abortion

Advanced Ultrasound in Diagnosis and Therapy 2019;03:123-127

DOI: 10.37015/AUDT.2019.190821

$\mathrm{C}$ ervical pregnancy is a rare type of ectopic pregnancy that implants in the endocervical canal. It accounts for less than $1 \%$ of all ectopic pregnancies [1]. Hysterectomy is the traditional treatment method for cervical pregnancy with severe complications. In order to maintain the reproductive capability of the patient, conservative treatment is preferred. However, the success of conservative treatment for cervical pregnancy depends on the initial diagnostic accuracy. Furthermore, early accurate diagnosis allows early intervention, which may increase the chances of successful preservation of reproductive capability. In this series, we describe the contrast-enhanced ultrasonography features of cervical pregnancy in contrast with inevitable abortion in the cervix, which, to our knowledge, has been rarely mentioned before.

\section{Case Reports}

There were 3 patients referred to our hospital for suspicion of cervical pregnancy. Contrast-enhanced ultrasonography (CEUS) was performed in order to make an accurate diagnosis. The study protocol was in keeping with the latest Helsinki declaration and was communicated to the Institutional Review Board of the hospital. All patients signed a consent form before examination of CEUS. SonoVue was used as ultrasound contrast agent (Bracco Suisse SA), which is a suspension of hexafluoride gas forming microbubbles with an average diameter of 2.5 micrometers (range from 0.5 up to 7.5 micrometers). The bubbles survive transpulmonary passage and recirculate, producing systemic ultrasound enhancement. A total of $2.4 \mathrm{ml}$ of contrast agent suspension $(29.5 \mathrm{mg}$ of Sulfur Hexafluoride) was administered to all patients through a peripheral vein, followed by a flush of 20 $\mathrm{ml}$ of sterile saline solution as per standard procedure. The enhancement phase was divided into the early enhancement phase and the late enhancement phase. The assessment was carried out for at least 3 min after contrast injection. CEUS was performed with a transvaginal curved array probe $(3-10 \mathrm{MHz})$.

\section{Case 1}

A 43-year-old woman, gravida 2 para 1 , admitted

\footnotetext{
* Corresponding Author: Department of Ultrasonography, West China Second University Hospital, Sichuan University, Chengdu, Sichuan Province 610041, China

e-mail: jessica_cd@163.com

2576-2508/O AUDT 2019 • http://www.AUDT.org

This is an open-access article distributed under the terms of the Creative Commons Attribution 4.0 International license, which permits unrestricted use, distribution and reproduction in any medium provided that the original work is properly attributed.
} 
to our hospital with mild painless vaginal bleeding following 66 days of amenorrhoea. Quantitative $\beta$-hCG level was $110163.9 \mathrm{mIU} / \mathrm{mL}$, and hemoglobin $11.3 \mathrm{~g} /$ dL. Transvaginal ultrasonography showed an empty uterine cavity and the presence of a gestational sac $(8.4 \times 5.4 \times 7.3 \mathrm{~cm})$ with a live embryo at the level of the cervix below the internal os. Crown-rump length was $29 \mathrm{~mm}$, embryo cardiac activity was present.

CEUS was then performed. The wall of the gestational sac showed rapid enhancement (10s after injection) during the early enhancement phase with the performance of hyper-enhanced, while the fetus was not enhanced. Enhancement sustained at a high level of intensity through the early enhancement phase, with the sign of doughnut-shaped in the wall of the gestational sac, and washed out slowly during the late enhancement phase. The enhancement of the cervix was posterior to gestational sac during the early enhancement phase, and the wash-out was prior to gestational sac during the late enhancement phase (Fig. 1). A diagnosis of cervical pregnancy was made.

Patient received conservative approach with a combination of methotrexate and uterine artery embolization and suction curettage. Blood loss during the operation was about $600 \mathrm{ml}$ and tamponade with foley catheter was performed. No blood transfusion was needed.

\section{Case 2}

A 29-year-old woman, gravida 1 para 0 , admitted to our hospital with vaginal bleeding following 42 days of amenorrhoea. Quantitative $\beta$-hCG level was 16708.0 $\mathrm{mIU} / \mathrm{mL}$, and hemoglobin $9.0 \mathrm{~g} / \mathrm{dL}$. Transvaginal ultrasonography showed an empty uterine cavity and the presence of a gestational sac $(3.1 \times 2.7 \times 2.5 \mathrm{~cm})$ at the level of the cervix below the internal os. No embryo or yolk sac was detected in the gestational sac.

CEUS was then performed. The wall of the gestational sac showed rapid enhancement (8s after injection) during the early enhancement phase with the performance of hyper-enhanced, enhancement sustained at a high level of intensity through the early enhancement phase, with the sign of doughnut-shaped in the wall of the gestational sac, and washed out slowly during the late enhancement phase. The enhancement of the cervix was posterior to gestational sac during the early enhancement phase, and the wash-out was prior to gestational sac during the late enhancement phase (Fig. 2). A diagnosis of cervical pregnancy was made.

Patient received conservative approach with a combination of methotrexate and suction curettage. Blood loss during the operation was about $100 \mathrm{ml}$. No blood transfusion was needed.
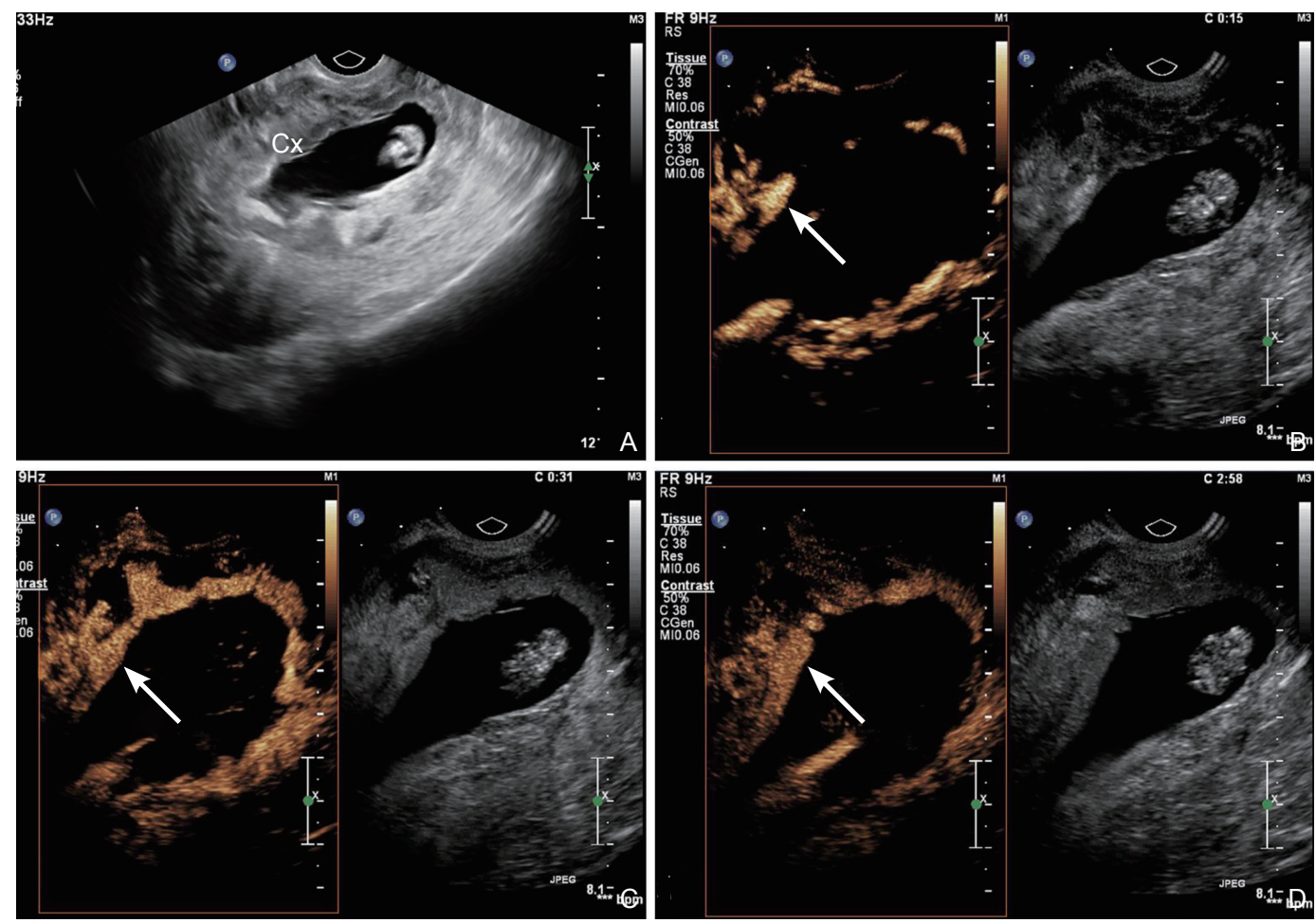

Figure 1 Ultrasonographic findings of Case 1. (A) Transvaginal ultrasonography showed the presence of a gestational sac with embryo at the cervix canal; (B) The right image shows the fundamental mode, and the left image shows the CEUS mode. The wall of the gestational sac showed rapid enhancement during the early enhancement phase with the performance of hyper-enhanced (white arrow); (C) Enhancement with the sign of doughnutshaped in the wall of the gestational sac (white arrow); (D) Contrast agents washed out slowly during the late enhancement phase (white arrow). 

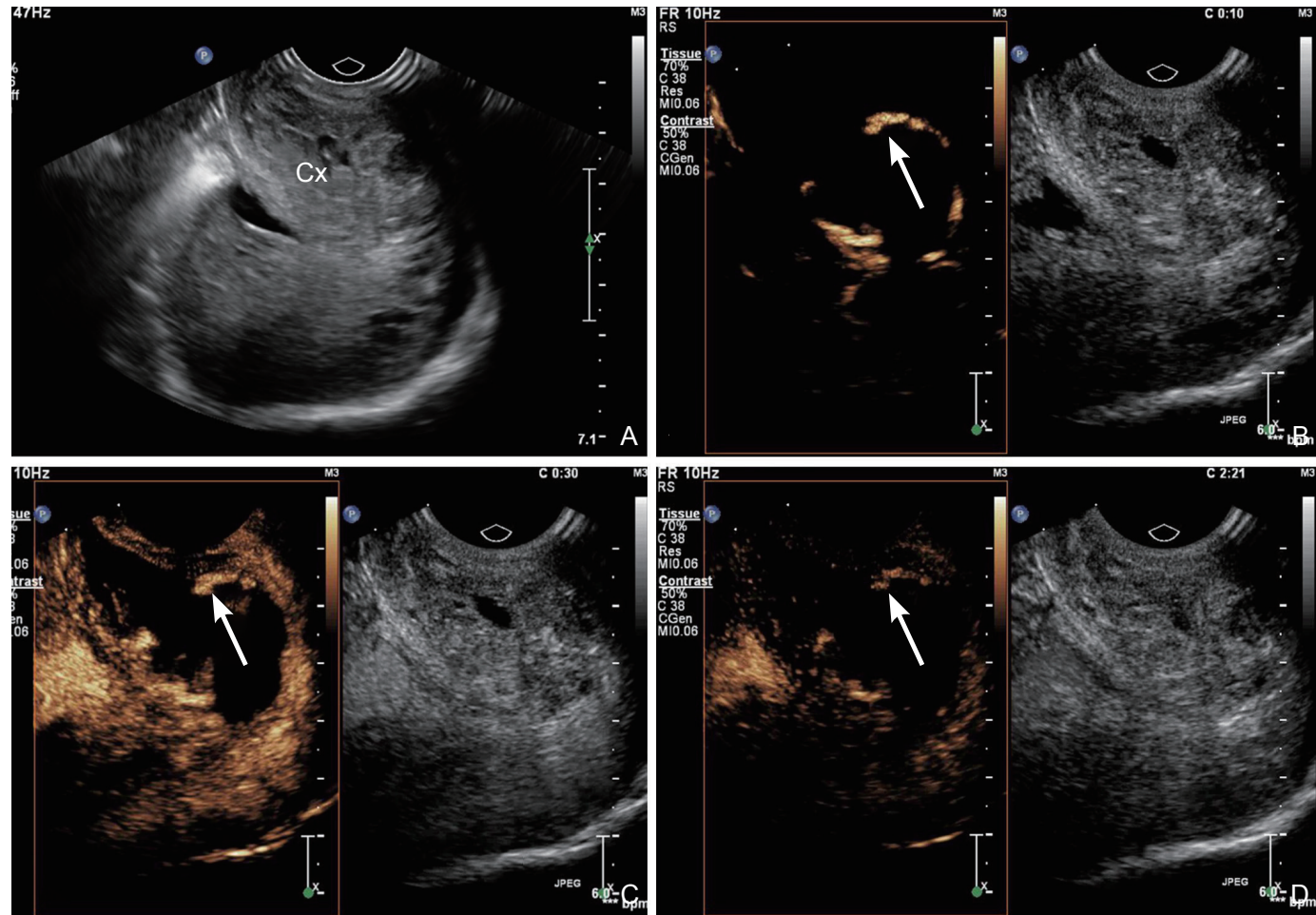

Figure 2 Ultrasonographic findings of Case 2. (A) Transvaginal ultrasonography showed a gestational sac at the cervix canal; (B) The right image shows the fundamental mode, and the left image shows the CEUS mode. The wall of the gestational sac showed rapid enhancement during the early enhancement phase with the performance of hyper-enhanced (white arrow). (C) Enhancement with the sign of doughnut-shaped in the wall of the gestational sac (white arrow); (D) Contrast agents washed out slowly during the late enhancement phase (white arrow).
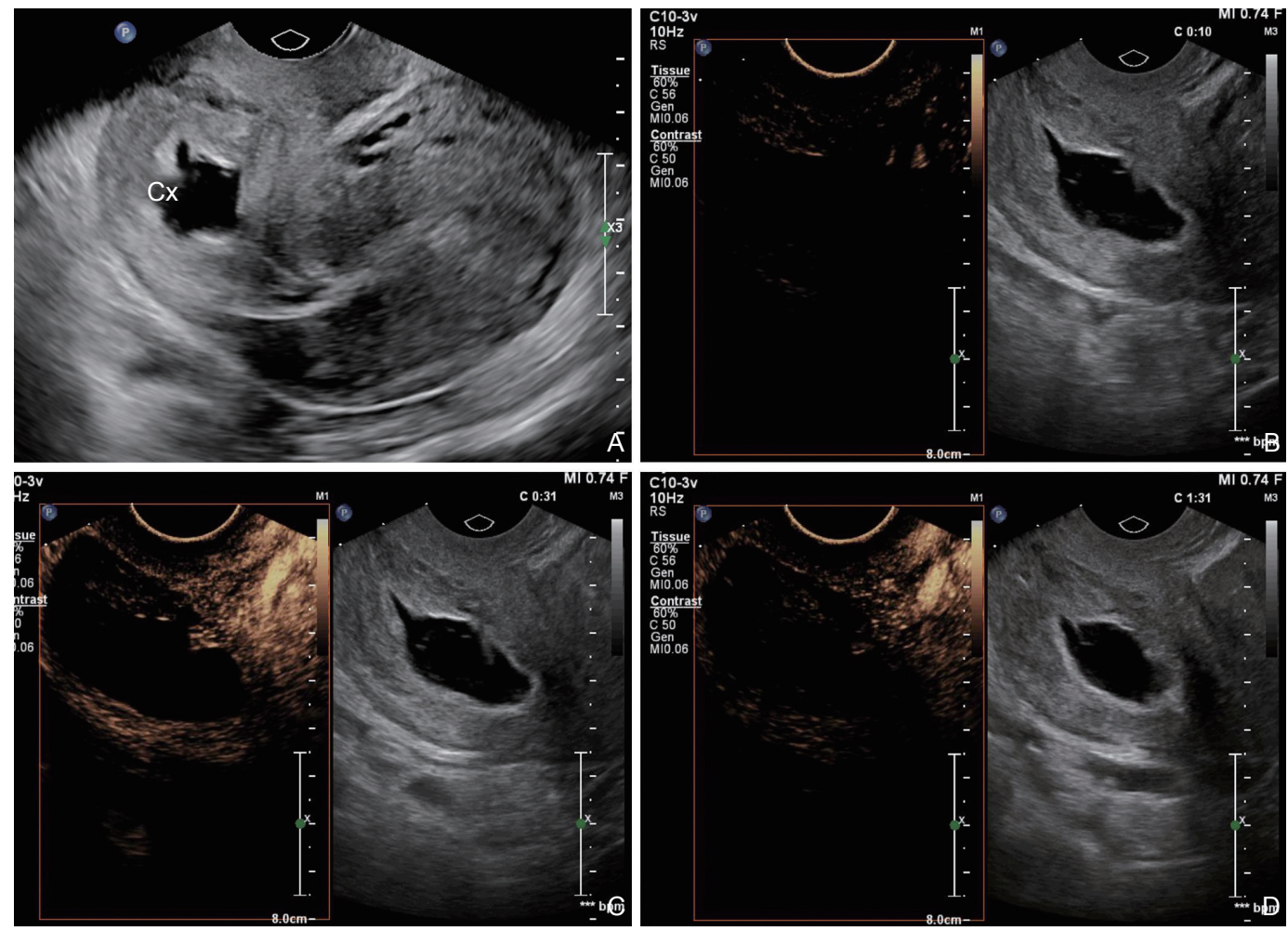

Figure 3 Ultrasonographic findings of Case 3. (A) Transvaginal ultrasonography showed a gestational sac at the cervix canal; (B) The right image shows the fundamental mode, and the left image shows the CEUS mode. The wall of the gestational sac showed no enhancement during the early enhancement phase; (C) No enhancement with the sign of doughnut-shaped in the wall of the gestational sac; (D) The wall of the gestational sac showed no enhancement during the late enhancement phase. 


\section{Case 3}

A 39-year-old woman, gravida 2 para 1, admitted to our hospital with vaginal bleeding following 40 days of amenorrhoea. Quantitative $\beta$-hCG level was 1594.0 $\mathrm{mIU} / \mathrm{mL}$, and hemoglobin $10.0 \mathrm{~g} / \mathrm{dL}$. Transvaginal ultrasonography showed an empty uterine cavity and the presence of a gestational sac $(4.2 \times 1.3 \times 1.8 \mathrm{~cm})$ at the level of the cervix below the internal os. No embryo or yolk sac was detected in the gestational sac.

CEUS was then performed. The wall of the gestational sac showed no enhancement during the early enhancement phase and late enhancement phase. The echogenicity of the cervix wall enhanced gradually during the early enhancement phase and late enhancement phase (Fig. 3). A diagnosis of inevitable abortion was made.

The patient underwent a conservative pregnancy termination procedure of evacuation. Blood loss during the operation was about $60 \mathrm{ml}$. No blood transfusion was needed.

\section{Discussion}

Cervical pregnancy is rare, and is often associated with significant morbidity. It may also have an effect on women's reproductive function. The cervix lacks the protective decidual plate seen in the corpus, which can block the invading of chorionic villi. Thus, the trophoblast can penetrate the cervical wall and into the uterine blood supply [2]. This may lead to massive bleeding, hemorrhagic shock and even death.

Before the advent of ultrasound, cervical pregnancy was not recognized clinically when the patient had vaginal bleeding following amenorrhoea, and was often misdiagnosed as threatened abortion or inevitable abortion. The diagnosis was often made after hysterectomy was done [3]. However, various conservative management modalities had been applied to preserve fertility in the recent past [4-6]. Early and accurate ultrasound diagnosis can increase the chances of successful conservative treatment for cervical pregnancy [7].

Raskin suggested [4] sonographic diagnostic criteria in 1978 as follows: enlargement of the cervix, uterine enlargement, diffuse amorphous intrauterine echoes and absence of intrauterine pregnancy [8]. Jurkovic et al proposed [2] additional diagnostic criteria in 1996 as "sliding sign" and peritrophoblastic blood flow to the conceptus by color Doppler flow [9]. Using these sonographic criteria, the accuracy of transvaginal ultrasound to detect cervical pregnancy was reported to be $87.5 \%[10]$.

CEUS is a well-established technique which uses ultrasound contrast agents to improve visualization and characterization of anatomic structure and lesions. It has been used in evaluating the blood perfusion of organs such as heart, liver, brain and kidneys. The size of ultrasound contrast agents is equal or smaller than red blood cells, which can survive transpulmonary passage and recirculate, producing systemic ultrasound enhancement. In our series, three patients underwent CEUS in addition to conventional sonography. Sonographic contrast agents that act as blood pool tracers have overcome the limitations of conventional sonography and enable imaging of parenchymal microvasculature [11]. In a true cervical pregnancy with placenta accrete lacking the cleavage plane, rich blood supply of trophoblast results in rapid enhancement of gestational sac wall during early enhancement phase of CEUS. On the contrary, CEUS shows no enhancement of the gestational sac in an aborting intrauterine pregnancy residing in the cervix wall because there is no blood supply from cervix.

We have retrospectively summarized the sonographic characteristics of cervical pregnancy in contrast with inevitable abortion in the cervix in three patients and estimated the value of CEUS in guiding clinical treatment. To our knowledge, a study using the contrast agent SonoVue for evaluation of cervical pregnancy has not been reported previously. We conclude that CEUS is more useful than conventional sonography for detection of cervical pregnancy because of the ability of SonoVue to perfuse microvessels. However, this result should be confirmed in a more in-depth study.

Sonographic examinations are valuable for diagnosis of cervical pregnancy and for guidance of clinical therapy. CEUS is better than conventional sonography for assessments of the lesion blood supply In spite of high diagnostic accuracy, CEUS also has several advantages including lack of radiation exposure [12], non-nephrotoxic contrast agents and lower cost. It can be the primary choice to early diagnose cervical pregnancy.

\section{Funding}

This work was supported by Sichuan Science and Technology Program (No. 2016JY0099 and 2018SZ0178) and by New sprout fund of West China Second University Hospital (No. kx091)

\section{Conflict of Interest}

The authors have no conflict of interest to declare.

\section{References}

[1] Bouyer J, Coste J, Fernandez H, Pouly JL, Job-Spira N. Sites of ectopic pregnancy: a 10 year population-based study of 1800 cases. Hum Reprod 2002;17:3224-30.

[2] Hosni MM, Herath RP, Mumtaz R. Diagnostic and therapeutic 
dilemmas of cervical ectopic pregnancy. Obstet Gynecol Surv 2014;69:261-76.

[3] anstede MM, van't Hof DB, van Groningen K, de Graaf IM. Severe complication after termination of a second trimester cervical pregnancy. Fertil Steril 2008;90:2009.e5-7.

[4] Bianchi P, Salvatori MM, Torcia F, Cozza G, Mossa B. Cervical pregnancy. Fertil Steril 2011;95:2123.e3-4.

[5] Yang JH, Shih JC, Liu KL, Yang YS. Combined treatment with temporary intraoperative balloon occlusion of common iliac arteries and hysteroscopic endocervical resection with postoperative cervical balloon for intractable cervical pregnancy in an infertile woman. Fertil Steril 2007;88:1438.e11-3.

[6] Vilos G, Abu-Rafea B, Kozak R. Safe resectoscopic evacuation of a 10 -week viable cervical pregnancy after transfemoral bilateral uterine artery embolization. Fertil Steril 2005;84:509.
[7] Vela G, Tulandi T. Cervical pregnancy: the importance of early diagnosis and treatment. J Minim Invasive Gynecol 2007;14:481-4.

[8] Raskin MM. Diagnosis of cervical pregnancy by ultrasound: a case report. Am J Obstet Gynecol 1978;130:234-5.

[9] Jurkovic D, Hacket E, Campbell S. Diagnosis and treatment of early cervical pregnancy: a review and a report of two cases treated conservatively. Ultrasound Obstet Gynecol 1996;8:373-80.

[10] Ushakov FB, Elchalal U, Aceman PJ, Schenker JG. Cervical pregnancy: past and future. Obstet Gynecol Surv 1997;52:45-59.

[11] Correas JM, Bridal L, Lesavre A, Méjean A, Claudon M, Hélénon O. Ultrasound contrast agents: properties, principles of action, tolerance, and artifacts. Eur Radiol 2001;11:1316-28.

[12] Hricak H, Brenner DJ, Adelstein SJ, Frush DP, Hall EJ, Howell RW, et al. Managing radiation use in medical imaging: a multifaceted challenge. Radiology 2011;258:889-905. 